\title{
Forensic Interviewers' Difficulty With Invitations: Faux Invitations and Negative Recasting
}

\author{
Hayden M. Henderson' ${ }^{(D,}$, Natalie Russo', and Thomas D. Lyon'
}

\begin{abstract}
An ongoing challenge for forensic interviewers is to maximize their use of invitations, such as requests that the child "tell me more about" details mentioned by the child. Examining 434 interviews with 4- to 12-year-old children questioned about abuse, this study analyzed (I) faux invitations, in which interviewers prefaced questions with "tell me" but then asked a noninvitation, (2) negative recasts, in which interviewers started to ask an invitation but then recast the question as a wh- or option-posing question, and (3) other aspects of questions that may relate to productivity independent of their status as invitations. About one fourth of "tell me" questions were faux invitations, and over $80 \%$ of recasts were negative. The frequency of both faux invitations and negative recasts increased during the substantive phase of the interviews, and these were related to decreased productivity, increased nonresponsiveness, and increased uncertainty. In contrast, use of exhaustive terms (e.g., "tell me everything") and nonstatic questions (e.g., about actions) was related to increased productivity. The results suggest that training should teach interviewers when and how strategic use of invitations and other question types can elicit specific types of forensically relevant information.
\end{abstract}

\section{Keywords}

children, forensic interview, invitations, training, productivity

Invitations are input-free prompts used to elicit recall responses from children (Lamb et al., 2018). They include questions that ask for additional recall about previously mentioned details, such as "What happened next?" A common approach to asking invitations is to use the phrase "tell me," coupled with "about," "everything about," or "more about," to invite the child to elaborate on previously mentioned information (e.g., "Tell me more about the hitting"). Invitations are distinguished from most wh- prompts (other than "what happened"), which also elicit recall, but which delimit the scope of the question (what, how, why, where, who, and when).

Research has demonstrated that invitations are the most effective type of prompt in eliciting productive (Lamb et al., 2018) and consistent responses (Brown et al., 2013) from children as young as 4 years of age (Lamb et al., 2003) throughout the course of the interview (Hershkowitz, 2001). Adoption of the national institute of child health and human development (NICHD) protocol, which emphasizes the utility of invitations, has been found to increase the successful prosecution of sexual abuse (Pipe et al., 2013). Wh- questions, which are often called directives, are less productive than invitations (Lamb et al., 2018). In turn, option-posing questions (yes/no and forcedchoice questions) are less productive than directive wh- questions and are also subject to greater error (Andrews et al., 2016). Thus, researchers recommend maximizing the use of invitations when interviewing child witnesses (Brubacher et al., 2019; Lamb et al., 2018; La Rooy et al., 2015; Lyon, 2014), as do the leading protocols and practice guidelines (American Professional Society on the Abuse of Children [APSAC], 2012; Lamb et al., 2018; Newlin et al., 2015).

However, interviewers typically fail to use invitations as often as they could (Lamb et al., 2018). Observational research has found that as few as $2 \%$ of interviewers' substantive questions are invitations (Lamb et al., 1996), but if interviewers are trained and receive feedback using the NICHD structured protocol, this percentage can be as high as $48 \%$ in the field (Cyr \& Lamb, 2009) and 65\% in experimental work (Brown et al., 2013).

We suspected that interviewers find it difficult to formulate and use invitations, particularly when they are seeking specific information. We tested this possibility in several ways. First, we examined the extent to which interviewers used faux invitations, defined as wh- prompts or option-posing questions that

\footnotetext{
'University of Southern California, Los Angeles, CA, USA

Corresponding Author:

Hayden M. Henderson, University of Southern California, 699 Exposition Blvd, Los Angeles, CA 90089, USA.

Email: hhenderson@law.usc.edu
} 
used the "tell me" stem common in true invitations. For example, "Tell me where it happened." Second, we examined recasting, defined as questions that begin as one type of question but are then rephrased as another type. Positive recasts occur when an interviewer rephrases a noninvitation as an invitation, and negative recasts occur when an interviewer rephrases an invitation as a noninvitation (i.e., as a wh- or option-posing question). For example, "Tell me what happened next. Did he hurt you?" is a negative recast. A high percentage of faux invitations and negative recasts would suggest that interviewers have difficulty in phrasing their questions as invitations. Our analyses included assessments of whether the types of questions asked varied during the presubstantive or substantive portion of the interviews. If interviewers' use of faux invitations and negative recasts increased during the substantive phase, when the allegations were discussed, this would support the hypothesis that their difficulty in asking invitations is greatest when they are seeking details of the allegations.

Third, we compared the quality of true invitations and faux invitations, positive recasting and negative recasting, by calculating the productivity of children's responses and the extent to which children were nonresponsive or uncertain. These analyses would determine how faux invitations and negative recasts were related to children's productivity; we predicted that they would be associated with lower productivity. Fourth, we examined other aspects of questions that might contribute to greater or lesser productivity and nonresponsiveness, but which are not captured by the distinction between invitations and noninvitations. Specifically, we looked at whether interviewers added exhaustive terms (such as "everything") to true and faux invitations, whether they scaffolded their true invitations (in order to explicitly reference specific aspects of the child's report), and whether they asked about static concepts (such as descriptions) or nonstatic concepts (such as actions) when they asked faux invitations. In what follows, we review evidence suggesting that interviewers may misunderstand guidance on best practices and be unable or reluctant to use that guidance in eliciting forensically significant information.

\section{True and Faux Invitations}

A superficial review of protocols and publications discussing best practice might lead one to the belief that use of the phrase "tell me" is the key to asking invitations. In the NICHD protocol, invitations are often phrased with the words "tell me," for example: "Tell me everything that happened" and "You mentioned [event, action, object]; Tell me more about that" (Lamb et al., 2007). Invitations prefaced with "tell me" are common in other practice guides as well (APSAC, 2012; Lyon, 2014; Newlin et al., 2015).

The Achieving Best Evidence in Criminal Proceedings guide, which provides guidance for practitioners who interview vulnerable witnesses, stated that "verbs like 'tell' and 'explain' are likely to be useful" in the free-narrative stage of eliciting a statement from children (Home Office, 2011). Oxburgh and colleagues (2010) argued that it is "pedagogically reasonable and good practice to suggest in training that questions which begin 'Tell,' 'Explain,' or 'Describe' are most likely to function as open questions and thus elicit longer responses" (p. 55). Wright and Powell (2006) noted that "repeating the phrase 'Tell me' over in their mind" helped forensic interviewers in learning to use open-ended questions (p. 321).

However, the words "tell me" are neither necessary nor sufficient for a prompt to be an invitation. As noted above, wh- questions using the term "what happened" are considered invitations, and they are widely recommended (APSAC, 2012; Lyon, 2014; Newlin et al., 2015). On the other hand, "tell me" combined with a wh- prompt other than "what happened" is not an invitation. For example, "tell me what he looks like" is a wh-prompt and not an invitation because it focuses on a description of the person. "Tell me the color of the car" is a wh- prompt with a hidden wh- because it is synonymous with "what color was the car?" Indeed, many of the "tell me" questions in the NICHD protocol interview are wh- prompts (e.g., "tell me why you came to talk to me"). "Tell me" questions can also be optionposing: "tell me if his clothes were on or off" and "tell me whether his clothes were on" are option-posing questions (forced-choice and yes/no questions, respectively) because they are synonymous with "were his clothes on or off?" and "were his clothes on?" We will refer to "tell me" questions that are directives or option-posing questions as faux invitations.

Contributing to the difficulty is that invitations may or may not be synonymous with "open-ended" questions, depending on the researcher and coding scheme (Oxburgh et al., 2010). It may be correct to assert that questions that begin with "tell me" are likely to be open-ended, but only if wh- prompts are considered open-ended. However, many researchers, such as Powell and her colleagues, reserve the term open-ended for questions that would qualify as invitations (Powell et al., 2013; Powell \& Snow, 2007). This can add to practitioners' confusion, because although they are universally advised to maximize their use of open-ended questions, they may be unsure of how those questions are best defined.

Some research supports the possibility that interviewers are often uncertain about what constitutes an invitation. Wolfman and colleagues (2016) found that $13 \%$ of the wh- questions in their sample ( $7 \%$ of all questions) began with "Tell me," leading the authors to speculate that interviewers "may mistakenly believe they are using more open-ended questions than they actually are" (p. 115). Yi and Lamb (2018), testing Korean police officers trained to use the NICHD protocol, found that trainees sometimes misclassified wh- prompts as invitations $(20 \%)$ and invitations as wh- prompts (10\%). Powell and colleagues (2013), studying research assistants, online forensic interviewing trainees, and police trainees ability to classify questions as invitations, found that the most common error was to overlook "what happened" questions as invitations (13\% of errors; what they called "open-ended"), and the third most common error was to overcall "tell me" questions as invitations (5\% of errors).

However, this research is only equivocal evidence that interviewers' fail to ask invitations in the field because they are 
unaware of the definition of invitations. The faux invitations in Wolfman and colleagues (2016) might have been deliberate directive questions inartfully phrased as "tell me" prompts. In Powell and colleagues' (2013) study, the overall error rate ranged from $14 \%$ to $20 \%$, so that the errors noted here were a very small proportion of the questions (13\% of errors would constitute $2-3 \%$ of all questions, and $5 \%$ of errors would constitute no more than $1 \%$ of all questions). Hence, the use of a faux invitation could be either an accidental or deliberate failure to use a true invitation.

\section{Positive and Negative Recasting}

In addition to the distinction between true and faux invitations, another potentially informative form of question that has largely been ignored in prior research is recasting. Recasting occurs when an interviewer asks a question, but before allowing the child to respond, recasts the question as a different type of question. To our knowledge, there is only one previous mention of this question form in the literature. Studying police interviews with adult suspects, Read et al. (2014) noted that interviewers would often combine invitations with a closed or specific question (e.g., "So what happened then, did you take your clothes off?"). Surprisingly, they categorized those questions as invitations ("open-ended" in their scheme), despite noting that interviewees tended to respond to the noninvitation portion of the question.

We focused on two types of recasting, which we call positive recasting and negative recasting. In positive recasting, an option-posing or wh- question is recast as an invitation (e.g., "Did you go to his house? Tell me what happened next"). In negative recasting, an invitation is recast as a wh- prompt or option-posing question (e.g., "Tell me about your clothes. Were they on or off?"). Positive recasting suggests that interviewers are reformulating their questions as invitations, whereas negative recasting suggests that interviewers are successfully formulating invitations but abandoning them in favor of more specific questions. Recasting may be more or less deliberate but in any case reflects a move toward either greater or less openness.

\section{Presubstantive Versus Substantive Questioning}

Interviewers are trained to elicit narratives about nonabusive events using invitations in the early portion of interviews in order to give children practice in providing elaborate reports of their experiences (Lamb et al., 2018; Lyon, 2014). It is only once the child is more comfortable and more talkative that the interviewer transitions to the substantive portion of the interview and discusses the allegations. Analyzing the types of questions during the presubstantive and substantive phases provides some insight into interviewers' potential difficulties in maximizing their use of invitations. If interviewers are capable of asking true invitations but feel compelled to abandon invitations when eliciting abuse details, then one would expect to see differences in question type across phases. If interviewers are more likely to use faux invitations and negative recasting during the substantive phase, this supports the proposition that they believe true invitations are insufficient to elicit forensically relevant details.

\section{Productivity of Different Question Types}

Because invitations have consistently been found to elicit more information than wh- questions, yes/no questions, and forcedchoice questions, one would expect to find that true invitations and positive recasting are related to higher productivity than faux invitations and negative recasting. Furthermore, the relation should not depend on phase; they should be related to greater productivity during both the presubstantive and substantive phases of the interviews. We measured productivity by calculating response word count, which has been found to correlate highly with more labor-intensive methods for counting details (Dickinson \& Poole, 2000). We also measured two qualities of responses that may undermine productivity: nonresponsiveness, which assesses whether the child failed to respond to the question (including answering "I don't know" or "I don't remember"), and uncertainty, which measures whether the child qualified his or her response with the use of a term signifying uncertainty (such as "I think" or "maybe" or "I don't know/remember" coupled with a substantive answer).

\section{Other Question Characteristics: Exhaustivity, Scaffolding, and Staticity}

In order to provide interviewers with the maximum amount of assistance in framing productive questions, it may be helpful to elaborate on the distinctions between invitations and wh- questions and identify sometimes-overlooked qualities of questions that may be related to productivity. The three that were considered in this study are exhaustivity, scaffolding, and staticity. Exhaustivity concerns the extent to which the question explicitly asks for all information. Both invitations and faux invitations can often be worded more or less exhaustively. For example, one can reword "tell me what happened" as "tell me everything that happened" to make the question exhaustive. Similarly, with a faux invitation such as "tell me about where it happened," one can reword it as "tell me everything about where it happened."

Exhaustivity has received limited attention in the literature on interviewing. The cognitive interview includes an instruction that the respondent should provide every detail, even if it doesn't seem important. We have only been able to identify one study examining the productivity of this instruction, and it found no effect (Milne \& Bull, 2002). However, an instruction at the beginning of the interview may be less likely to increase productivity than an exhaustive request built into a question; the instruction seems more likely to be forgotten (or inaccessible) at the time specific questions are answered. Hence, explicitly referencing "everything" may be more productive. 
Scaffolding refers to the extent that when requesting elaboration, the interviewer provides guidance to the child regarding what the child should elaborate. Scaffolding may make true invitations more specific, and thus reduce the perceived need to add a wh- clause, which turns a true invitation into a faux invitation. For example, an unscaffolded invitation would be simply asking a child to "tell me more." It might be more productive to ask the child to "tell me more about [information previously generated by the child]." Scaffolding might be particularly helpful in reducing the likelihood that a child will be nonresponsive when asked an invitation.

Staticity refers to whether a what/how question is static (asks for a description; e.g., "what color was the car?") or nonstatic (asks about actions, causes, and evaluations; e.g., "what did he do?"). Research has shown that static what/how questions are negatively related to productivity in court (Andrews et al., 2016) and in forensic interviews (Ahern et al., 2018), and one study found that invitations that asked about appearances and locations (static) were related to less productive responses than invitations asking about actions (nonstatic; Lamb et al., 2003). We examined whether these differences also applied to faux invitations.

\section{This Study}

This study examined the prevalence and productivity of invitations (true and faux) and recasts (positive and negative) in forensic interviews and considered whether their prevalence and apparent productivity changed in comparing the presubstantive and substantive phase of the interviews. In addition, in order to assess whether additional qualities of questions were correlated with greater productivity, we considered whether true and faux invitations were exhaustive, whether true invitations were scaffolded, and whether faux invitations using "what" and "how" were static or nonstatic.

We hypothesized the following: (1) Interviewers ask a nontrivial number of faux invitations. (2) Interviewers ask a nontrivial number of negative recasts, in which they reword an invitation as a noninvitation. (3) The relative frequency of faux invitations and negative recasts increases during the substantive phase of the interviews. (4) True invitations are associated with longer responses than faux invitations. (5) Positive recasts are associated with longer responses than negative recasts. (6) Exhaustive true and faux invitations are associated with longer responses than nonexhaustive true and faux invitations. (7) Scaffolded true invitations are associated with longer responses than nonscaffolded true invitations. (8) Static what/how faux invitations are associated with longer responses than nonstatic what/how faux invitations.

\section{Method}

\section{Sample}

We examined 434 forensic interview transcripts. The interviews were conducted between 2004 and 2013 at one of five different Child Advocacy Center sites in Southern California.
Most of the interviewers would have received the California Forensic Interview Training, a statewide program that provides interviewers the 10-Step Protocol (Lyon, 2014), a revision of the NICHD protocol that incorporates questions from the protocol. For example, Step 6 includes narrative practice (e.g., "tell me everything that happened on your last birthday"), Step 7 recommends "tell me why you came to talk to me" as an initial allegation question, Step 8 recommends "tell me everything that happened" as an allegation follow-up, and Step 9 recommends "tell me more" and "what happened next" questions as additional follow-ups. However, the training focuses on the need to avoid recognition questions as much as the utility of invitations, interviewers are encouraged to tailor their approach to suit their needs, and the training is not equipped to provide ongoing supervision and refresher training.

The interviews had been transcribed and anonymized for training purposes, with the consent of the parent or legal guardian, and the archived data were used for the current study. Interviews were excluded if the interview was conducted in Spanish or if the child fell outside the age range. Participants were $71 \%$ female $(n=307)$ and $29 \%$ male $(n=127)$, ranging in age from 4 to 12 years, with a mean age of $7.5(S D=2.6)$. For analyses, children were categorized into three age groups: 4- to 6-year-olds $(41 \%, n=178), 7$ - to 9-year-olds $(33 \%$, $n=142)$, and 10 - to 12 -year-olds $(26 \%, n=114)$. The 7 - to 9-year old age-group was set as the baseline group in analyses. Children were alleged victims of sexual abuse $(97.7 \%$, $n=424)$, alleged victims of physical abuse $(1.4 \%, n=6)$, or a witness to alleged abuse $(0.9 \%, n=4)$.

\section{Coding of Transcripts}

All questions using the words "tell me" were identified and were coded as true or faux invitations. True invitations requested free recall about a topic mentioned by the child (e.g., "tell me what happened" and "tell me" coupled with a detail previously disclosed by the child). Faux invitations contained a wh- question word that narrowed the information requested (e.g., "tell me when it happened") or an optionposing yes/no or forced-choice question (e.g., "tell me if it was dark"). Recasts were questions that were originally worded as one question type (invitation or noninvitation), and then, before the child was given a chance to respond, worded as a different type. Specifically, positive recasts were noninvitations reworded as a true invitation, and negative recasts were invitations reworded as a noninvitation. Summaries of the coding categories for true and faux invitations and for positive and negative recasts are in Table 1 .

Interviews were separated into presubstantive and substantive phases. The presubstantive phase was the portion of the interview before the transition to the substantive phase, defined as the first question likely intended to elicit the allegation (e.g., "tell me why you came to see me"), or the first mention of the allegation by the child, whichever came first. The substantive phase included the transition and everything thereafter. The presubstantive phase typically 
Table I. Interviewers' Invitation Types.

\begin{tabular}{|c|c|c|}
\hline Prompt & Definition & Example \\
\hline True invitation & $\begin{array}{l}\text { A request for recall that asks } \\
\text { about what "happened" } \\
\text { or asks for additional } \\
\text { information about child- } \\
\text { generated information }\end{array}$ & $\begin{array}{l}\text { "Tell me everything } \\
\text { that happened" } \\
\text { "You said [child's } \\
\text { statement]; tell me } \\
\text { more about that" }\end{array}$ \\
\hline Faux invitation & $\begin{array}{l}\text { Interviewer asks child a } \\
\text { "wh-" or option-posing } \\
\text { prompt beginning with } \\
\text { "tell me" }\end{array}$ & $\begin{array}{l}\text { "Tell me about what } \\
\text { he did" } \\
\text { "Tell me whether his } \\
\text { clothes were on or } \\
\text { off" }\end{array}$ \\
\hline Positive recast & $\begin{array}{l}\text { Utterance that asked a } \\
\text { noninvitation, followed by } \\
\text { an invitation }\end{array}$ & $\begin{array}{l}\text { "Where did he go... } \\
\text { what happened after } \\
\text { that?" }\end{array}$ \\
\hline $\begin{array}{c}\text { Negative } \\
\text { recast }\end{array}$ & $\begin{array}{l}\text { Utterance that asked an } \\
\text { invitation, followed by a } \\
\text { noninvitation }\end{array}$ & $\begin{array}{l}\text { "Tell me everything } \\
\text { that happened, were } \\
\text { you scared?" }\end{array}$ \\
\hline
\end{tabular}

included introductions, interview instructions, rapportbuilding, and practice narratives.

Exhaustivity was determined by noting and categorizing the term following the words "tell me," and included an "everything" term (e.g., "tell me everything/all"), a "more about" term (e.g., "tell me more/something else about X"), no term (e.g., "tell me about"), or an "other" term (e.g., "tell me again"). Due to their infrequency ( $n=115)$, "other" terms were excluded from analyses. True invitations were further coded as scaffolded (explicit reference to information the child had previously provided either before or after the invitation; e.g., "you said it hurt; tell me more" "you said it hurt?"/“yes"/ "tell me more," or "tell me more about it hurting") or unscaffolded (e.g., "tell me more" or unelaborated pronoun, e.g., "tell me more about that"). Faux invitations with embedded "what" and "how" questions were classified as static or nonstatic. Summaries of the coding categories for exhaustivity, scaffolding, and staticity are in Table 2.

Children's productivity was measured by word count, omitting stuttered words within an utterance. Children's nonresponsiveness (e.g., no response, I don't know/not sure, request for clarification) and uncertainty (e.g., substantive response qualified by "I don't know" or "I'm not sure") were also coded and included as binary dependent variables.

\section{Interrater Reliability}

An independent reliability coder recoded $20 \%$ of the prompts. Interrater reliability coefficients for all variables were high, Kappa $(K)>0.80$. Agreement regarding the classification of true and faux invitations, $K=.97(S E=.02), 95 \%$ confidence interval (CI) [.93, 1.0]; negative recasts, $K=.89(S E=.05)$, $95 \%$ CI $[.79, .99]$; positive recasts, $K=.82(S E=.03), 95 \%$ CI $[.76, .88]$; exhaustivity term, $K=.98(S E=.01), 95 \%$ CI [.96, 1.0]; scaffolding, $K=.92,(S E=.02), 95 \%$ CI $[.88, .96]$; faux invitation subtypes, $K=.90(S E=.02), 95 \%$ CI [.86, .94]; faux invitation wh-subtypes, $K=.98(S E=.02), 95 \%$ CI [.94, 1.0]; children's nonresponsiveness $K=.85$ ( $S E=.01), 95 \%$ CI [.83, $.87]$; and children's uncertainty $K=.83(S E=.03), 95 \% \mathrm{CI}$ $[.77, .89]$ were all sufficient. Children's productivity (i.e., word count) was machine-calculated and therefore did not require a reliability score.

\section{Analysis Plan}

First, we present descriptive results regarding the proportion of true/faux invitations and positive/negative recasting. Second, we examine whether the proportion varied by interview phase. Third, we assess the different question types' productivity (in word count), children's nonresponsiveness, and children's uncertainty, taking into account children's age (4- to 6-year-olds, 7- to 9-yearolds, and 10- to 12-year-olds). Fourth, we conduct similar analyses examining exhaustivity, scaffolding, and staticity.

Analyses were conducted using generalized linear mixedeffects models (GLMMs). Fixed effects included interview phase (presubstantive and substantive), child's age (4-6 years old, 7-9 years old, and 10-12 years old), invitation type (true and faux), recasting type (positive and negative), and other question characteristics (exhaustivity ["everything," "more about," or none], scaffolding [scaffolded and unscaffolded], and staticity [static and nonstatic]). Dependent response variables included word count, nonresponsiveness, and uncertainty. All GLMM models included a by-subject (i.e., "child") random intercept to control for both the different number and types of questions addressed to each child as well as children's individual response proclivities. In the Poisson GLMMs (i.e., word count), an observation-level random effect was included to control for overdispersion; however, this was not necessary for the binary models (e.g., nonresponsiveness and uncertainty; Gardner et al., 1995). For example:

$$
\begin{aligned}
& \text { Word count } \sim \text { Invitation }+ \text { Child's Age }+ \text { Invitation } \\
& \times \text { Child's Age }+(1 \mid \text { child })+(1 \mid \text { observation })
\end{aligned}
$$

or

$$
\begin{aligned}
& \text { Nonresponsiveness } \sim \text { Invitation }+ \text { Child's Age }+ \text { Invitation } \\
& \times \text { Child's Age }+(1 \mid \text { child })
\end{aligned}
$$

Analyses were performed using the glmer function in the $\mathrm{R}$ package lme 4 with the bobyqa optimizer and Laplace approximations (Bates et al., 2015). GLMMs combine the properties of linear mixed models (which incorporate random effects) and generalized linear models (which handle nonnormal data) and are preferable to traditional analysis of variance models because they have fewer assumptions, handle response variables from different distributions (e.g., binary, count, or proportion), and maximize power while simultaneously estimating between-subject variance (Bates et al., 2015; Bolker et al., 2009; Pinhero \& Bates, 2000). The most complex converged models are reported below accompanied by the unstandardized fixed-effect estimates $(\beta)$, standard errors of the estimates $(S E)$, and estimates of significance ( $Z$ and $p$ values). Only significant 
Table 2. Interviewers' Other Question Type Characteristics.

\begin{tabular}{|c|c|c|c|}
\hline Prompt & Prompt Subtype & Definition & Example \\
\hline \multirow[t]{2}{*}{$\begin{array}{l}\text { Exhaustivity } \\
\qquad \text { (true and faux) }\end{array}$} & "Tell me everything" & $\begin{array}{l}\text { Interviewer uses a term after "tell me" that encourages } \\
\text { the child to tell everything or all }\end{array}$ & $\begin{array}{l}\text { "Tell me everything that } \\
\text { happened when you saw him" }\end{array}$ \\
\hline & “Tell me” no elaboration & Interviewer invites child to elaborate without more & "Tell me about his clothes" \\
\hline \multirow[t]{2}{*}{ Scaffolding (true) } & Scaffolded invitation & $\begin{array}{l}\text { A true invitation that includes explicit reference to information } \\
\text { previously generated by the child }\end{array}$ & $\begin{array}{l}\text { "Tell me more about [previously } \\
\text { generated information by the } \\
\text { child]" }\end{array}$ \\
\hline & Unscaffolded invitation & $\begin{array}{l}\text { A true invitation that either includes no scaffolding or only } \\
\text { an unelaborated pronoun (e.g., "that") }\end{array}$ & "Tell me more" \\
\hline Staticity $^{\mathrm{a}}$ (faux) & What/how static & $\begin{array}{l}\text { Questions that asked for non-action-related descriptions, } \\
\text { usually asking for contextual information such as location, } \\
\text { time, or objects }\end{array}$ & "Tell me what time it was" \\
\hline
\end{tabular}

${ }^{\text {a }}$ See Andrews et al. (2016) for more thorough definitions and examples.

findings $(p<.05)$ are reported descriptively in the Results section but adjusted means and output from all GLMM analyses can be found in Supplemental Appendices 1-6.

\section{Results}

\section{Frequency of True/Faux Invitations and Positive/Negative Recasts}

There were a total of 87,085 interviewer utterances $(M=201)$ and 7,723 prompts that included the term "tell me." The majority of "tell me" prompts were true invitations $(76 \%, n=5,873)$. Of the faux invitations $(n=1,850)$, the majority were "wh-" prompts $(97 \% ; n=1,797)$, and less than $3 \%(n=53)$ were option-posing prompts. There were 1,074 recasts. The majority of recasts were negative recasts $(80 \%, n=856)$, in which the interviewer recast an invitation as a noninvitation.

In the presubstantive phase, $86 \%(n=1,705)$ of the "tell me" prompts were true invitations, and $74 \%(n=191)$ of the recasts were negative recasts. In sum, $8 \%$ of the utterances were "tell me" invitations or noninvitations recast as invitations ( $M=4$ utterances per interview). In the substantive phase, $73 \%$ $(n=4,168)$ of the "tell me" prompts were true invitations, and $82 \%(n=665)$ of the recasts were negative recasts. In sum, $7 \%$ of the utterances were "tell me" invitations or noninvitations recast as invitations ( $M=10$ utterances per interview).

We examined the relation between interview phase and the frequency of true/faux invitations and recasts. The analyses included interview phase as a fixed effect and prompt type (true/faux; positive/negative recast) as dependent binary variables. Faux invitations were more common in the substantive phase (adjusted $M=29 \%$ ) than in the presubstantive phase (adjusted $M=13 \% ; B=-0.99, S E=0.08, Z=-12.87$, $p<.001)$. Negative recasts were more common in the substantive phase (adjusted $M=92 \%$ ) than in the presubstantive phase (adjusted $M=85 \% ; B=-0.68, S E=0.23, Z=-2.90, p=$ .004; see Supplemental Appendix 1).

Preliminary analyses revealed that interview phase did not significantly affect the interpretation of results regarding children's productivity, nonresponsiveness, or uncertainty. Thus, interview phase was subsequently excluded.

\section{True and Faux Invitations}

Analyses examined the relation between true and faux invitations and children's responses. The analyses included invitation type (true, faux), child's age (4-6 years old, 7-9 years old, and 10-12 years old), and child's age $\times$ invitation type as fixed effects. There were main effects of question type and age. As predicted, true invitations were associated with more productive responses $(M=12.33$ words) than faux invitations $(M=$ $8.28 ; B=0.40, S E=0.05, Z=8.74, p<.001)$. The 4- to 6year-old children were the least productive $(M=6.71 ; B=$ $-0.50, S E=0.09, Z=-5.77, p<.001)$. True invitations also elicited fewer nonresponsive $(7 \% ; B=-0.53, S E=0.15, Z=$ $-3.55, p<.001)$ and uncertain responses $(8 \% ; B=-0.52, S E$ $=0.14, Z=-3.81, p<.001$ ) than faux invitations (nonresponsive $=10 \%$; uncertain $=12 \%$ ). There were no other significant main or interaction effects (see Supplemental Appendix 2).

\section{Recasting}

Analyses examined the relation between interviewers' positive and negative recasts and children's responses. The analysis included recast type (positive and negative), child's age (4-6 years old, 7-9 years old, and 10-12 years old), and child's 
age $\times$ recast type as fixed effects. There were main effects of question type and age. As predicted, negative recasts were associated with shorter responses $(M=7.92$ words $)$ than positive recasts $(M=13.78, B=0.57, S E=0.15, Z=3.69$, $p<.001)$. The 4 - to 6 -year-old children were the least productive $(M=7.12, B=-0.47, S E=0.13, Z=-3.75, p<.001)$ and the most nonresponsive $(10 \% ; B=0.82, S E=0.31$, $Z=2.64, p=.01)$. There were no other significant main or interaction effects (see Supplemental Appendix 3).

\section{Exhaustivity}

Analyses examined the relation between exhaustivity and children's responses. The analysis included exhaustivity terms ("everything," "more about," or none), child's age (4-6 years old, 7-9 years old, and 10-12 years old), and child's age $\times$ exhaustivity terms as fixed effects. Consistent with our hypotheses, the failure to use an exhaustivity term was associated with briefer responses $(M=10.03$ words, $B=-0.21, S E=0.05, Z$ $=-4.14, p<.001)$ and the use of "everything" was associated with the longest responses $(M=14.79, B=0.18, S E=0.06, Z$ $=3.15, p<.001)$. The 4 - to 6 -year-old children were the least productive $(M=7.92, B=-0.55, S E=0.09, Z=-6.08, p<$ $.001)$ and the 10- to 12 -year old children were the most productive $(M=16.80, B=0.20, S E=0.10, Z=2.14, p=.03)$. There were no other significant main or interaction effects on children's responses (see Supplemental Appendix 4).

\section{Scaffolding}

Analyses explored the relation between interviewers' scaffolding and children's responses to true invitations. The analysis included scaffolding (scaffolded and unscaffolded), child's age (4-6 years old, 7-9 years old, and 10-12 years old), and child's age $\times$ scaffolding as fixed effects. There were main effects of age only. The 4- to 6-year-old children were the least productive $(M=7.87$ words, $B=-0.62$, $S E=0.11, Z=-5.45, p<.001)$. There were no other significant main or interaction effects on children's responses (see Supplemental Appendix 5).

\section{What/How Staticity}

Supplemental Appendix 6a details the different types of faux wh- invitations, specifying the wh- word used and, for the what/how questions, whether the questions were static or nonstatic. Analyses explored the relation between interviewers' static and nonstatic faux invitation prompts and children's responses. The analysis included staticity (static and nonstatic), child's age (4-6 years old, 7-9 years old, and 10-12 years old), and child's age $\times$ staticity as fixed effects. There were main effects of question type and age. As predicted, static prompts elicited less productive responses ( $M=4.79$ words) than nonstatic prompts $(M=9.99, B=-0.71, S E=0.15, Z=-4.74$, $p<.001)$. The 4- to 6-year-old children were the least productive $(M=4.74, B=-0.48, S E=0.11, Z=-4.44, p<.001)$.
There were no other significant main or interaction effects on children's responses (see Supplemental Appendix 6).

\section{Discussion}

This study examined the extent to which interviewers failed to ask true invitations in forensic interviews, and whether their tendency to do so appeared to be influenced by a need to elicit specific details. We assessed how often interviewers asked faux invitations: questions that began with "tell me" and thus looked like invitations, but which were actually wh- or option-posing questions. We also assessed recasts: questions that began as invitations but then were rephrased as noninvitations (negative recasts) or vice versa (positive recasts). We tested whether the proportion of true and faux invitations and positive and negative recasts changed from the presubstantive to the substantive phase of the interviews.

We found that most "tell me" questions were true invitations (76\%), and only a very small percentage of "tell me" questions were option-posing questions (3\%). This suggests that interviewers know how to ask invitations. However, we also found that most recasts were negative $(80 \%)$ and that both faux invitations and negative recasts were more common in the substantive than in the presubstantive phases of the interviews. Indeed, the proportion of faux invitations more than doubled, and the proportion of positive recasts fell by more than half. This suggests that when interviewers are seeking specific information about the allegations they are more likely to forsake true invitations.

This study also assessed the quality of different types of questions by examining productivity, nonresponsiveness, and uncertainty. We found that true invitations were associated with more productive responses than faux invitations, and positive recasts appeared more productive than negative recasts, both in the presubstantive and substantive phases of the interview. We also found that true invitations elicited fewer nonresponsive answers and uncertain answers. Although younger children were consistently less productive than older children, the question type differences were not influenced by age, which means that younger children appeared to be as likely to benefit from true invitations as older children.

This study also assessed other qualities of questions that are not captured by the distinction between invitations and whquestions. We found that productivity was greater when interviewers used exhaustive terms ("everything" compared to "more about," "more about" compared to "about") and when their what/how questions were nonstatic (e.g., asked about actions rather than descriptions). We did not find, however, that scaffolding was associated with productivity or responsiveness, that is, whether interviewers explicitly referenced children's prior statements when asking invitations.

The study has a number of limitations. First, as with almost all observational work, a limitation of the study is that children's reports could not be verified. Hence, it is unclear whether more productive answers are as accurate as less productive answers. Second, the study cannot establish precisely 
why interviewers ask faux invitations or negative recasts. We suspect that interviewers often believe that they are asking invitations but are insufficiently aware either of the definition of invitations or of the form of their questions. However, it is also possible that interviewers are well aware of the definition of invitations and that their frequent use of faux invitations reflects inartful phrasing of specific questions rather than misunderstanding. That is, they may be deliberately asking directive questions but phrasing them as faux invitations or negative recasts. Similarly, negative recasting may sometimes reflect the use of invitations as topic introductions rather than aborted attempts to use invitations to elicit substantive information. Below, we discuss the implications of the different explanations for training.

Third, because we did not manipulate the use of different question types, causality could not be proven. For example, we cannot say with certainty that had interviewers substituted faux invitations or negative recasts with true invitations, they would have obtained more productive responses. Interviewers might have sensed that children's free recall was exhausted when they asked faux invitations or recast questions as more direct. In support of this possibility, one could point to the fact that the average interview in this sample included 10 substantive "tell me" invitations (and an unspecified number of "what happened" invitations) and that this is not much lower than some research on the NICHD protocol (e.g., Cyr \& Lamb, 2009, finding protocol interviews contained 21 invitations). However, examining raw numbers is potentially misleading because NICHD protocol interviews tend to have fewer questions, and as a percentage of all questions, invitations are much more prevalent (48\%; Cyr \& Lamb, 2009).

\section{Implications for Training and Future Research}

Interviewers who narrow their questions in the hopes of eliciting specific information should be made aware that this risks reducing children's productivity, responsiveness, and certainty. The frequent use of faux invitations, particularly during the substantive portion of the interviews, suggests that interviewers may need explicit guidance that "tell me" questions are not the same as invitations and that any wh- word necessarily narrows the focus of a question, unless one is asking "what happened." Furthermore, interviewers should be warned not to pair "tell me" with "if" or "whether" because this signals a yes/no question. Consistent with research identifying the benefits of refutational training, in which common misconceptions are first described and then refuted (Tippett, 2010), it may be helpful to give interviewers explicit examples of faux invitations and explain why they are not true invitations. If interviewers asking faux invitations demonstrate awareness that they are failing to ask true invitations, but believe that children's free recall has been exhausted, then they should be encouraged to persist in asking invitations throughout the interview. Training can also highlight the advantages of exhaustivity in questions (in particular the advantage of references to "everything") and the greater productivity of nonstatic questions (particularly references to actions).

Whatever the cause of faux invitations, training should also include guidance on how true invitations can elicit specific types of information. For example, we have found that "tell me more" invitations about specific portions of children's narrative are useful in eliciting details that children have omitted, often because of apparent embarrassment or shame. The interviewer asks the child to "tell me more" about the portion of the narrative containing the missing detail. Similarly, we have also found that invitations using the word "happened" can also elicit otherwise omitted details. For example, asking about "what happened last" often elicits details about the completion of the abuse. Future research should explore the productivity of different types of invitations in eliciting specific types of information, and how training can best convince interviewers of the utility of invitations.

At the same time that interviewers can be encouraged to maximize their use of invitations, training can acknowledge that wh- questions are effective in eliciting certain types of information that children tend not to provide when answering invitations. For example, children often omit relevant details when asked free recall questions, such as their subjective reaction to abuse (Lyon et al., 2012; McWilliams et al., in press; Newman \& Roberts, 2014), details regarding abuse disclosure (Malloy et al., 2013), and prior conversations (Stolzenberg et al., 2018). Nonstatic questions such as "how did people find out about [the abuse]," "how did you feel [during/after abuse]," and "what did [suspect] say about [the abuse]" may be utilized when invitations fail to elicit these types of information. Future research can explore the extent to which invitations elicit or fail to elicit other types of forensically important information, and what types of wh- questions are most productive.

The finding that exhaustive questions were associated with longer responses than nonexhaustive questions could be further explored with respect to specific types of wh- questions. For example, in developmental work, young children have been found to have a nonexhaustive interpretation of "who" questions. If asked, "who is holding a ball" and shown a picture depicting a number of people, several of which are holding a ball, young children will often point to only one person (Roeper et al., 2007). An unexamined question is whether children's nonexhaustive interpretation of "who" appears in forensic interviews and whether it can be overcome through exhaustive questions, such as "tell me everyone who..." Furthermore, whether other wh- questions are similarly affected has not been tested. It may be the case that asking, "tell me everything you felt" is more productive than "how did you feel," and will elicit multiple subjective reactions from children who would otherwise report only one reaction.

In sum, this study examined true and faux invitations and positive and negative recasts and showed how forensic interviewers questioning children about abuse frequently cast or recast their questions in order to elicit specific information, especially during the substantive phase of their interviews. In doing so, they may lose the advantages of asking invitations, 
potentially reducing the productivity, responsiveness, and certainty of children's responses. Furthermore, the study identified other aspects of questions beyond the invitation/ noninvitation distinction that appears to increase productivity, namely the use of exhaustive terminology and nonstatic questions. The challenge is to train interviewers in how to maximize their use of open-ended questions without sacrificing their need for specific details.

\section{Acknowledgments}

The authors would like to thank Anya Hee and Hailey Konovalov for their invaluable assistance with this project.

\section{Declaration of Conflicting Interests}

The author(s) declared no potential conflicts of interest with respect to the research, authorship, and/or publication of this article.

\section{Funding}

The author(s) disclosed receipt of the following financial support for the research, authorship, and/or publication of this article: This study was supported in part by the National Institute of Child Health and Development (Grant HD087685), the Social Sciences and Humanities Research Council of Canada, and the Canadian Foundation for Innovation.

\section{ORCID iD}

Hayden M. Henderson (D) https://orcid.org/0000-0003-4652-7839

\section{Supplemental Material}

Supplemental material for this article is available online.

\section{References}

Ahern, E. C., Andrews, S. J., Stolzenberg, S. N., \& Lyon, T. D. (2018). The productivity of wh- prompts in child forensic interviews. Journal of Interpersonal Violence, 33, 2007-2015. https:// doi.org/10.1177/0886260515621084

Andrews, S. J., Ahern, E. C., Stolzenberg, S. N., \& Lyon, T. D. (2016). The productivity of wh- prompts when children testify. Applied Cognitive Psychology, 30, 341-349. https://doi.org/10.1002/ acp.3204

American Professional Society on the Abuse of Children. (2012). Practice guidelines: Forensic interviewing in cases of suspected child abuse. https://www.apsac.org/guidelines

Bates, D., Mächler, M., Bolker, B., \& Walker, S. (2015). Fitting linear mixed-effects models using lme4. Journal of Statistical Software, 67, 1-48. https://doi.org/10.18637/jss.v067.i01

Bolker, B. M., Brooks, M. E., Clark, C. J., Geange, S. W., Poulsen, J. R., Stevens, M. H., \& White, J. S. (2009). Generalized linear mixed models: A practical guide for ecology and evolution. Trends in Ecology \& Evolution, 24, 127-135. https://doi.org/10.1016/j.tree. 2008.10.008

Brown, D. A., Lamb, M. E., Lewis, C., Pipe, M., Orbach, Y., \& Wolfman, M. (2013). The NICHD investigative interview protocol: An analogue study. Journal of Experimental Psychology: Applied, 19, 367-382. http://dx.doi.org/10.1037/a0035143

Brubacher, S. P., Peterson, C., La Rooy, D., Dickinson, J. J., \& Poole, D. A. (2019). How children talk about events: Implications for eliciting and analyzing eyewitness reports. Developmental Review, 51, 70-89. https://doi.org/10.1016/j.dr.2018.12.003

Cyr, M., \& Lamb, M. E. (2009). Assessing the effectiveness of the NICHD investigative interview protocol when interviewing French-speaking alleged victims of child sexual abuse in Quebec. Child Abuse \& Neglect, 33, 257-268.

Dickinson, J., \& Poole, D. A. (2000). Efficient coding of eyewitness narratives: A comparison of syntactic unit and word count procedures. Behavior Research Methods, Instruments, \& Computers, 34, 537-545. https://doi.org/10.3758/BF03200826

Gardner, W., Mulvey, E. P., \& Shaw, E. C. (1995). Regression analyses of counts and rates: Poisson, overdispersed poisson, and negative binomial models. Psychological Bulletin, 118, 392-404. http://dx.doi.org.libproxy2.usc.edu/10.1037/0033-2909.118.3.392

Hershkowitz, I. (2001). Children's responses to open-ended utterances in investigative interviews. Legal and Criminological Psychology, 6, 49-63. https://doi.org/10.1348/135532501168190

Home Office. (2011). Achieving the best evidence in criminal proceedings: Guidance on interviewing victims and witnesses, and guidance on using special measures. http://www.cps.gov.uk/publi cations/docs/best_evidence_in_criminal_proceedings.pdf

La Rooy, D., Heydon, G., Korkman, J., \& Myklebust, T. (2015). Interviewing child witnesses. In G. Oxburgh, T. Myklebust, T. Grant, \& R. Milne (Eds.), Communication in investigative and legal contexts: Integrated approaches from forensic psychology, linguistics and law enforcement (pp. 57-78). John Wiley.

Lamb, M. E., Brown, D. A., Hershkowitz, I., Orbach, Y., \& Esplin, P. W. (2018). Tell me what happened (2nd ed.). Wiley.

Lamb, M. E., Hershkowitz, I., Sternberg, K. J., Boat, B., \& Everson, M. D. (1996). Investigative interviews of alleged sexual abuse victims with and without anatomical dolls. Child Abuse \& Neglect, 20, 1251-1259.

Lamb, M. E., Orbach, Y., Hershkowitz, I., Esplin, P. W., \& Horowitz, D. (2007). A structured forensic interview protocol improves the quality and informativeness of investigative interviews with children: A review of research using the NICHD investigative interview protocol. Child Abuse \& Neglect, 31, 1201-1231. https:// doi.org/10.1016/j.chiabu.2007.03.021

Lamb, M. E., Sternberg, K. J., Orbach, Y., Esplin, P. W., Stewart, H., \& Mitchell, S. (2003). Age differences in young children's responses to open-ended invitations in the course of forensic interviews. Journal of Consulting and Clinical Psychology, 71, 926-934. http://dx.doi.org/10.1037/0022-006X.71.5.926

Lyon, T. D. (2014). Interviewing children. Annual Review of Law \& Social Science, 10, 73-89. https://doi.org/10.1146/annurev-law socsci-110413-030913

Lyon, T. D., Scurich, N., Choi, K., Handmaker, S., \& Blank, R. (2012). "How did you feel?": Increasing child sexual abuse witnesses' production of evaluative information. Law and Human Behavior, 36, 448-457. http://dx.doi.org/10.1037/h0093986

Malloy, L. C., Brubacher, S. P., \& Lamb, M. E. (2013). "Because she's one who listens" children discuss disclosure recipients in forensic interviews. Child Maltreatment, 18, 245-251. https://doi.org/10. 1177/1077559513497250

McWilliams, K., Stolzenberg, S. N., Williams, S., \& Lyon, T. D. (in press). Increased maltreated and nonmaltreated children's recall 
disclosures of a minor transgression: The effects of back-channel utterances, a promise to tell the truth and a post-recall putative confession. Child Abuse \& Neglect.

Milne, R., \& Bull, R. (2002). Back to basics: A componential analysis of the original cognitive interview mnemonics with three age groups. Applied Cognitive Psychology, 16, 743-753. https:// doi.org/10.1002/acp.825

Newman, J. E., \& Roberts, K. P. (2014). Subjective and nonsubjective information in children's allegations of abuse. Journal of Police \& Criminal Psychology, 29, 75-80. https://doi.org/ 10.1007/s11896-013-9133-y

Newlin, C., Steele, L., Chamberlain, A., Anderson, J., Kenniston, J., Russell, A., Stewart, H., \& Vaughan-Eden, V. (2015). Child forensic interviewing: Best practices. US Department of Justice, Office of Justice Programs, Office of Juvenile Justice and Delinquency Prevention.

Oxburgh, G., Myklebust, T., \& Grant, T. (2010). The question of question types in police interviews: A review of the literature from a psychological and linguistic perspective. International Journal of Speech, Language and the Law: Forensic Linguistics, 17, 45-66. https://doi.org/10.1558/ijsll.v17i1.45

Pinhero, J. C., \& Bates, D. M. (2000). Linear mixed-effects models: Basic concepts and examples. In J. C. Pinhero \& D. M. Bates (Eds.), Statistics and computing mixed-effects models in S and S-PLUS (pp. 3-52). Springer. https://doi.org/10.1007/0-387-22747-4_1

Pipe, M. E., Orbach, Y., Lamb, M. E., Abbott, C. B., \& Stewart, H. (2013). Do case outcomes change when investigative interviewing practices change? Psychology, Public Policy, and Law, 19, 179-190.

Powell, M. B., Benson, M. S., Sharman, S. J., Guadagno, B., \& Steinberg, R. (2013). Errors in the identification of question types in investigative interviews of children. International Journal of Police Science \& Management, 15, 144-156. https://doi.org/ 10.1350/ijps.2013.15.2.308
Powell, M. B., \& Snow, P. C. (2007). Guide to questioning children during the free-narrative phase of an investigative interview. Australian Psychologist, 42, 57-65. https://doi.org/10.1080/ 00050060600976032

Read, J., Powell, M., Kebbell, M., Milne, B., \& Steinberg, R. (2014). Evaluating police interviewing practices with suspects in childsexual abuse cases. Policing and Society, 24, 523-544. https:// doi.org/10.1080/10439463.2013.784297

Roeper, T., Schulz, P., Pearson, B. Z., \& Reckling, I. (2007). From singleton to exhaustive: The acquisition of Wh. Proceedings of Semantics of Understudied Languages 2005 Conference. Buffalo NY, pp. 87-102. http://people.umass.edu/roeper/611/ wh-specificity\%2005.pdf

Stolzenberg, S. N., McWilliams, K., \& Lyon, T. D. (2018). Children's conversational memory regarding a minor transgression and a subsequent interview. Psychology, Public Policy, \& Law, 24, 379-392. http://dx.doi.org/10.1037/law0000176

Tippett, C. D. (2010). Refutation text in science education: A review of two decades of research. International Journal of Science and Mathematics Education, 8, 951-970. https://doi.org/10.1007/ s10763-010-9203-x

Wolfman, M., Brown, D., \& Jose, P. (2016). Talking past each other: Interviewer and child verbal exchanges in forensic interviews. Law and Human Behavior, 40, 107-117. http://dx.doi.org/10.1037/ lhb0000171

Wright, R., \& Powell, M. B. (2006). Investigative interviewers' perceptions of their difficulty in adhering to open-ended questions with child witnesses. International Journal of Police Science \& Management, 8, 316-325. https://doi.org/10.1350/ijps. 2006.8.4.316

Yi, M., \& Lamb, M. E. (2018). How accurately do police officers identify the types of questions used in investigative interviews with child victims? Korean Journal of Forensic Psychology, 9, 117-135. 